\title{
Erratum: Charged Point Defects in the Flatland: Accurate Formation Energy Calculations in Two-Dimensional Materials [Phys. Rev. X 4, 031044 (2014)]
}

\author{
Hannu-Pekka Komsa, Natalia Berseneva, Arkady V. Krasheninnikov, and Risto M. Nieminen
}

(Received 25 April 2018; revised manuscript received 18 June 2018; published 16 August 2018)

DOI: 10.1103/PhysRevX.8.039902

The correction method presented in the original paper involves calculating the electrostatic energy difference between model systems where charge is placed in (i) a small supercell, of the same size as used in the density functional theory (DFT) calculations, and subject to periodic boundary conditions $E_{\text {periodic }}$, and (ii) isolated charge $E_{\text {isolated }}$. We proposed that the latter can be efficiently obtained by extrapolation from the former. While conceptually true, the extrapolations presented in the original paper were not evaluated from sufficiently large supercell sizes, which led to underestimation of the correction. In this Erratum, we briefly analyze the origin of the error, show how to circumvent it, and provide corrected energy values.

The reason for this oversight was that, unlike in bulk systems with a homogeneous dielectric constant, the electrostatic energy for 2D systems shows a sudden change in the asymptotic behavior at large supercell sizes, evidencing the existence of two characteristic length scales. This is illustrated in Fig. 1, which shows the extrapolation of $E_{\text {periodic }}$ as a function of the inverse of the supercell size scaling parameter $\alpha$ in the case of the $\mathrm{Mn}_{\mathrm{Mo}}(+1)$ defect in $\mathrm{MoS}_{2}$. Here, $\alpha=1$ corresponds to a $4 \times 4$ supercell and $c=12 \AA$, and all cell dimensions increase uniformly upon increasing $\alpha\left(L_{x, y, z} \rightarrow \alpha L_{x, y, z}\right)$. In the original paper, the extrapolation was performed using a second-order polynomial with respect to $1 / \alpha$, fitted to energies from the five smallest supercell sizes, and it was found to result in a good fit (cf. the dashed-dotted red line and solid circles in Fig. 1). For $1 / \alpha \lesssim 0.25$, corresponding to supercells larger than $16 \times 16$, there is a sudden upturn. This issue was first pointed out by Noh et al. [1] (and later on reported at least in Refs. [2,3]), who suggested using a fifth-order polynomial for the fitting.

From the data shown in Fig. 1 and the higher-order polynomial fits, it is still not clear if they reproduce the correct extrapolated value. On the other hand, an efficient method for calculating $E_{\text {isolated }}$ was recently presented in Ref. [4] that

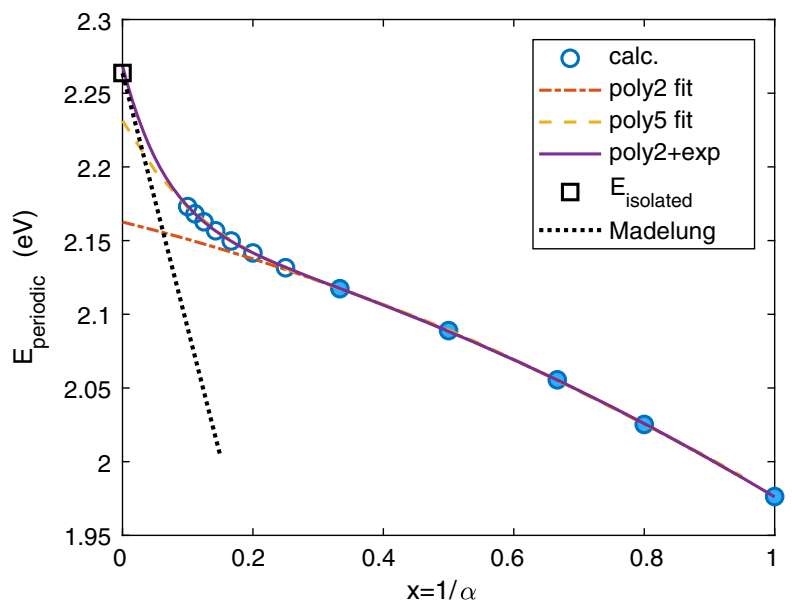

FIG. 1. Energy of the periodic system $E_{\text {periodic }}$, as a function of the inverse supercell scaling parameter $\alpha$. Only the first five calculated data points (marked by filled circles) were used for fitting of the second-order polynomial.

Published by the American Physical Society under the terms of the Creative Commons Attribution 3.0 License. Further distribution of this work must maintain attribution to the author(s) and the published articles title, journal citation, and DOI. 
TABLE I. Special vacuums $L_{z}^{\mathrm{s}}$ (in $\AA$ ) for different lateral supercell sizes (in multiples of the primitive cell) of monolayer $h$-BN and $\mathrm{MoS}_{2}$.

\begin{tabular}{lllllllr}
\hline \hline System & $4 \times 4$ & $5 \times 5$ & $6 \times 6$ & $7 \times 7$ & $8 \times 8$ & $9 \times 9$ & $10 \times 10$ \\
\hline$h$-BN & 18.37 & 24.12 & 30.27 & 36.73 & 43.46 & 50.41 & 57.56 \\
MoS $_{2}$ & 18.45 & 22.88 & 27.58 & 32.55 & 37.78 & 43.27 & 49.04 \\
\hline \hline
\end{tabular}

TABLE II. Formation energies and charge transition levels for all considered defects in $2 \mathrm{D} h$-BN and $\mathrm{MoS}_{2}$ (in eV). For formation energies of charged defects, the Fermi level is set to the valence band maximum. Charge transition levels are referenced to the valence band maximum. Substitutional defect formation energies are taken from the extrapolation, whereas adatom energies are taken from the special vacuum calculation with a $6 \times 6$ supercell. Three dots denote cases where the relevant charge state is unstable.

\begin{tabular}{lccccc}
\hline \hline Defect & $E^{f}\left[X^{0}\right]$ & $E^{f}\left[X^{+1}\right]$ & $E^{f}\left[X^{-1}\right]$ & $\varepsilon(0 /+1)$ & $\varepsilon(0 /-1)$ \\
\hline $\mathrm{C}_{\mathrm{B}}$ & 1.66 & -0.59 & $\ldots$ & 2.25 & $\ldots$ \\
$\mathrm{C}_{\mathrm{N}}$ & 4.28 & $\ldots$ & 6.50 & $\ldots$ & 2.20 \\
$\mathrm{C}_{\mathrm{ad}}$ & 6.65 & 6.58 & 9.05 & 0.07 & 2.40 \\
$\mathrm{~B}_{\mathrm{ad}}$ & 8.15 & 6.98 & 11.46 & 1.16 & 3.31 \\
$\mathrm{~N}_{\mathrm{ad}}$ & 4.62 & 5.10 & $\cdots$ & -0.48 & 1.58 \\
$\mathrm{Mn}_{\mathrm{Mo}}$ & 1.91 & 1.22 & $\cdots$ & 0.68 & $\cdots$ \\
$\mathrm{F}_{\mathrm{S}}$ & -1.73 & -2.65 & & 0.92 & $\cdots$ \\
\hline \hline
\end{tabular}

eliminates the need for extrapolation. It relies on Bessel expansion in lateral directions and a truncated Green's function approach in the perpendicular direction. It can be readily generalized to an anisotropic dielectric tensor profile and to a charge density comprised of several Gaussians with different widths $\sigma$ in the lateral and perpendicular directions. The value obtained with this approach is found to reside $30 \mathrm{meV}$ higher than the fifth-order polynomial fit (black square in Fig. 1).

While direct evaluation of $E_{\text {isolated }}$ should usually be preferred, if an extrapolation procedure is nevertheless needed, one can make use of the fact that the energies at $x=1 / \alpha \rightarrow 0$ simply correspond to the Madelung energy $E_{\mathrm{M}}$ of a point charge in a homogeneous background charge and no screening $(\varepsilon=1)$. The lack of screening is clear for interactions between charges in different sheets, going largely through vacuum. While the interactions between charges in the same sheet are partially screened, at increasing distance, the screening becomes weaker as a larger fraction of the field lines pass through the vacuum, and the effective interaction approaches that of an unscreened one [5]. This change in the screening is the cause for the two length scales. One could then fit only the energies in the weak screening regime with a low-order polynomial, if such large supercells are computationally tractable [3]. Alternatively, we found that the energies can be well fitted in both regimes when the second-order polynomial is appended with an exponential term:

$$
E=c_{0}+c_{1} x+c_{2} x^{2}+d e^{-c_{3} x}
$$

where $c_{i}$ are the fitting parameters, and $d=\left(c_{1}-\partial E_{\mathrm{M}} / \partial x\right) / c_{3}$ guarantees that the energy gradient is similar to the Madelung energy at $x=1 / \alpha \rightarrow 0$, i.e., $\partial E / \partial x=\partial E_{\mathrm{M}} / \partial x$. As seen in Fig. 1, this works better than the fifth-order polynomial with the given number of data points reaching supercell sizes of $40 \times 40$. Naturally, as more data points are calculated, the extrapolations from different functions should converge. Similarly, good agreement with the $E_{\text {isolated }}$ and the extrapolations using Eq. (1) were obtained in all cases, giving us confidence that we indeed have reached the correct value for $E_{\text {isolated. }}$. Finally, we note that the Madelung energy scaled with the average dielectric constant yields fairly good corrected values.

The magnitude of errors induced by this are (i) $0.185 \mathrm{eV}$ in the case of $\mathrm{C}_{\mathrm{B}}$ (and adatoms $\mathrm{C}_{\mathrm{ad}}, \mathrm{B}_{\mathrm{ad}}, \mathrm{N}_{\mathrm{ad}}$ at +1 and -1 charge states via the special vacuum construction) in $h$ - $\mathrm{BN}$; (ii) $0.177 \mathrm{eV}$ in the case of $\mathrm{C}_{\mathrm{N}}$ in $\mathrm{h}-\mathrm{BN}$; (iii) $0.101 \mathrm{eV}$ in the case of $\mathrm{Mn}_{\mathrm{Mo}}$ in $\mathrm{MoS}_{2}$; and (iv) $0.099 \mathrm{eV}$ in the case of $\mathrm{F}_{\mathrm{S}}$ in $\mathrm{MoS}_{2}$. Correspondingly, a rigid shift should be applied to all corrected values in Figs. 2-4, both formation energies and charge transition levels. Here, it is sufficient to present revised versions of Tables I and II. While the errors are non-negligible, they are relatively small when compared to the calculated band gaps of these materials: $4.67 \mathrm{eV}$ for $h-\mathrm{BN}$ and $1.69 \mathrm{eV}$ for $\mathrm{MoS}_{2}$. As a result of this shift, extrapolations from DFT values using a second-order polynomial will not match the corrected values, due to the same problems as discussed above. 


\section{ACKNOWLEDGMENTS}

We thank fruitful discussions with Damien West. We thank the Academy of Finland for the support under Projects No. 286279 and No. 311058. We also thank the CSC-IT Center for Science Ltd and the Aalto Science-IT project for generous grants of computer time.

[1] J.-Y. Noh, H. Kim, and Y.-S. Kim, Stability and Electronic Structures of Native Defects in Single-Layer MoS 2 , Phys. Rev. B 89, 205417 (2014).

[2] M. H. Naik and M. Jain, Coffee: Corrections for Formation Energy and Eigenvalues for Charged Defect Simulations, Comput. Phys. Commun. 226, 114 (2018).

[3] T. Cao and A. Bongiorno, Atomistic Corrective Scheme for Supercell Density Functional Theory Calculations of Charged Defects, Sci. Rep. 7, 2834 (2017).

[4] R. Sundararaman and Y. Ping, First-Principles Electrostatic Potentials for Reliable Alignment at Interfaces and Defects, J. Chem. Phys. 146, 104109 (2017).

[5] P. Cudazzo, I. V. Tokatly, and A. Rubio, Dielectric Screening in Two-Dimensional Insulators: Implications for Excitonic and Impurity States in Graphane, Phys. Rev. B 84, 085406 (2011). 